\title{
Investigation of Magnetic Field Interharmonics and Sideband Vibration in the FSCW IPMSM Drive With the SPWM Technique
}

\author{
Wenyi Liang, Patrick Chi-Kwong Luk ${ }^{\circledR}$, Senior Member, IEEE, and Weizhong Fei, Member, IEEE
}

\begin{abstract}
The high-frequency sideband current harmonics are the inherent concomitants of applying sinusoidal pulse width modulation (SPWM) in permanent-magnet synchronous machine drives. Abundant spatial interharmonic magnetic field components in fractional-slot concentrated-winding (FSCW) interior permanent-magnet synchronous machine (IPMSM) render it very susceptible to electromagnetic radial vibration. In this paper, a universal analytical interharmonic model of magnetic field in FSCW IPMSMs is proposed, and further, it is employed to develop the sideband radial force density model of SPWM. Both comprehensive finite-element analysis (FEA) simulations and experimental tests are carried out to underpin the validity of the analytical models. The close agreements between the analytical, FEA, and experimental results have revealed that the proposed analytical approaches can deliver accurate sideband current and vibration predictions with minimum computational efforts and suffice for fast preliminary assessments. In addition, the validated analytical models can offer detailed and insightful revelations of various factors that affect the corresponding sideband vibration components.
\end{abstract}

Index Terms-Concentrated winding, electromagnetic radial force density, electromagnetic radial vibration, fractional slot, magnetic flux density, permanent-magnet synchronous machine (PMSM) drive, sideband harmonic, sinusoidal pulse width modulation (SPWM).

\section{NOMENCLATURE}

$\alpha_{v}$

$\alpha_{s}$

$b, b_{0}$

$b_{a}^{p}, b_{a}^{v}$

$b_{\mu}^{v}, b_{\mu}^{v}$

$B_{0}$
$B_{a}^{p}, B_{a}^{v}$

$B_{\mu}^{p}, B_{\mu}^{v}$

$\delta$

$f, f_{\kappa}$

$F_{\kappa v}, \theta_{\kappa}$

$F_{p}, F_{v}$

$i_{d}, i_{q}$

$i_{\kappa}$

$K_{w p}, K_{w v}$

$L_{a d}, L_{a q}$

$L_{d}, L_{q}$

$L_{\text {ef }}$

$\lambda$

$\Lambda_{v}$

$\Lambda_{\delta}, \Lambda_{s l}$

$M$

$N$

$\omega_{1}, \omega_{\kappa}$

$\omega_{s}$

$p$

$p_{r}$

$\psi_{f}$

$\theta$

$\theta_{s}$

$\tau_{p}$

$\tau_{s}$

$U_{\mathrm{dc}}$

Z
Fundamental and $v$-order armature reaction flux density amplitudes.

Fundamental and $v$-order sideband armature reaction flux density amplitudes.

Torque angle.

Overall and $\omega_{\kappa}$-frequency MMF.

Amplitude and initial phase of $v$-order $\omega_{\kappa}$ frequency MMF.

Amplitudes of fundamental and $v$-order MMF.

$d$ and $q$ components of the stator current vector.

$\kappa$-order current harmonic.

Fundamental and $v$-order winding coefficient.

$d$ and $q$ fundamental components of armature reaction inductances.

$d$ and $q$ inductance components.

Effective length of a motor.

Permeance of the machine.

$v$-order average air gap permeance.

Air gap, PM groove permeance.

Modulation index.

Number of serial-connected turns per phase.

Fundamental and $\kappa$-order angular speed.

Carrier angular speed.

Pole pairs of a machine.

Radial force density.

PM flux linkage.

Mechanical angle.

Initial phase of the current vector.

Pole arc distance of a fundamental component.

PM pole arc coefficient.

Direct current bus voltage.

Slot number of a machine.

\section{INTRODUCTION}

Manuscript received October 18, 2016; revised January 31, 2017 and April 29, 2017; accepted May 26, 2017. Date of publication June 1, 2017; date of current version January 3, 2018. The work was supported by the Engineering and Physics Science Research Council, U.K., under Grants Ref.EP/I038543/1 and Ref.EP/L001063/1. Recommended for publication by Associate Editor L. Dalessandro. (Corresponding author: Patrick Chi-Kwong Luk.)

The authors are with the Centre of Power Engineering, Cranfield University, Cranfield MK43 0AL, U.K. (e-mail: w.liang@cranfield.ac.uk; p.c.k.luk@ cranfield.ac.uk; w.fei@cranfield.ac.uk).

Color versions of one or more of the figures in this paper are available online at http://ieeexplore.ieee.org.

Digital Object Identifier 10.1109/TPEL.2017.2711062
$\mathbf{T}$ $\mathrm{HE}$ fractional-slot concentrated-winding (FSCW) permanent-magnet synchronous machines (PMSMs) have been gaining attention in various application due to their distinctive advantages [1]. However, the rich spatial interharmonics, introduced by the armature reaction from the peculiar winding configuration, will induce parasitic characteristics, such as vibration and acoustic noise. Many investigations have been carried out to study the interharmonic components and associated characteristics. The MMF distribution is numerically 
studied for a concentrated winding machine in [2]. Various stator design techniques such as stator magnetic flux barrier and new winding arrangements are proposed to reduce MMF harmonics in FSCW PMSMs [3]-[6]. Meanwhile, the resultant air-gap magnetic field components associated with armature MMF interharmonics in the inset PMSM is analytically investigated in [7]. A rotor pole with a sinusoidal profile is implemented to reduce the harmonics of the air-gap magnetic field and hence further improve the vibration and noise behavior[8]. The air gap is partially enlarged to smooth the radial stress in the air gap to reduce the vibration in the machine [9]. The harmonic leakage inductance of outer rotor FSCW PMSMs is investigated by involving the winding factors [10], [11]. Such rich air-gap magnetic field components will induce electromagnetic radial force components with various spatial orders, which are prone to excite significant electromagnetic radial vibration and acoustic noise in the machine [12]-[15].

There have been very limited studies on the analytical investigation of interharmonic magnetic field in FSCW interior permanent-magnet synchronous machines (IPMSMs). The existing research studies are mainly concentrated on the numerical analysis of the magnetic field and its associated vibrations in FSCW IPMSMs [16]-[18]. More importantly, they all only focus on the low-frequency components contributed by the permanent magnets (PMs) and stator armature reaction. There is still a dearth of analytical investigations on high-frequency vibration and noise associated with pulse width modulation (PWM) techniques. The existing studies on such high-frequency characteristics are mainly devoted to induction machine drive systems [19]-[21]. Not until quite recently have there been particular investigations on full direct analytical derivations of sideband vibration [22] at PMSM drive systems with the space-vector pulse width modulation (SVPWM) technique, based on the analytical sideband current model [23], [24]. The discussion is only confined to integral-slot configuration, and the method cannot be directly extended to cover FSCW configuration [22]. The vibration model in FSCW PMSMs is far more complex due to the abundant interharmonics introduced by the peculiar winding configuration. Therefore, an effective model to analytically estimate the interharmonic components becomes imperative for analytical investigation of vibration in such a machine.

The sideband current model from SVPWM [23] is extended to embrace sinusoidal pulse width modulation (SPWM) in this paper. A new interharmonic magnetic field model is also developed for the FSCW machine. As a result, a closed-form analytical model is accomplished for sideband radial force in FSCW IPMSMs. In Section II, the universal analytical derivation of the critical average air-gap permeance is carried out for FSCW IPMSMs. Section III is dedicated to the general model of interharmonic magnetic field components based on the new permeance model. In Section IV, the main sideband current components are identified by an analytical model, and associated magnetic field and radial force density components are presented. Section V focuses on the experimental validations of sideband current and vibration based on a ten-pole 12-slot prototype, followed by the concluding remarks in Section VI.

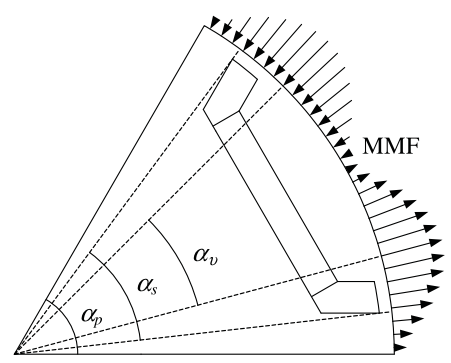

(a)

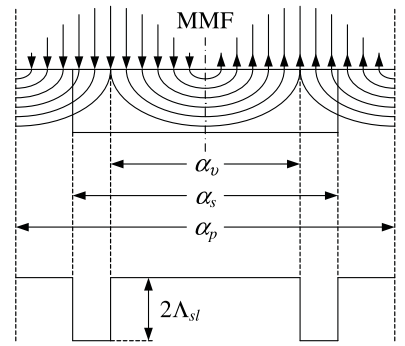

(b)
Fig. 1. Demonstration of magnetic circuit and its permeance. (a) Rotor structure of a PMSM. (b) Magnetic circuit and its permeance.

\section{AVERAGE PERMEANCE OF MMF INTERHARMONICS IN FSCW IPMSMS}

\section{A. Armature Reaction and Associated Permeance}

The air-gap magnetic field introduced by the armature reaction can be expressed as

$$
b(\theta, t)=f(\theta, t) \cdot \lambda(\theta, t) .
$$

Besides the MMF components in the integral-slot machine, there are abundant of MMF interharmonic components. Certain interharmonic components are quite close to and sometimes even higher than the fundamental component, typically the $(Z-p)$ order in double-layer-winding machines and extra $t_{0}$-order in single-layer-winding machines, where $t_{0}$ is the greatest common divisor (GCD) of stator slot number $Z$ and rotor pole pair number $p$. The universal MMF model of the $\omega_{\kappa}$-frequency harmonic current can be expressed as

$$
f_{\kappa}(\theta, t)=\sum_{v=1} F_{\kappa v} \cos \left(v \theta-\omega_{\kappa} t-\theta_{\kappa}\right)
$$

where the amplitude of the $v$-order MMF component is determined by

$$
F_{\kappa v}=\frac{3 N K_{w v} i_{\kappa}}{v \pi} .
$$

$v$ is the spatial order number related to the pole-slot combination and winding arrangement. Generally, the slot harmonics are the main components in FSCW PMSMs. The frequency $\omega_{\kappa}$ can be either positive or negative, which represents the rotational direction of the magnetic field with reference to the fundamental component.

It can be observed from (1) that the average magnetic permeance $\Lambda_{0}$ is the predominant component for interharmonics in FSCW PMSMs, similar to the fundamental magnetic field component in [22]. For the sake of simplicity, only this part is taken into account for the permeance analysis. However, $\Lambda_{0}$ is dependent on the corresponding spatial order of the harmonic component and the torque angle in IPMSMs. The complex magnetic flux paths in the peculiar rotor structure make it a variable. Hence, a new permeance model is studied in this part. The rotor structure of a common single-layer IPMSM is depicted in Fig. 1(a), while the magnetic flux path under $v$-order MMF harmonic is demonstrated in Fig. 1(b). Apparently, the mean value of permeance is spatial order dependent due to the potential 


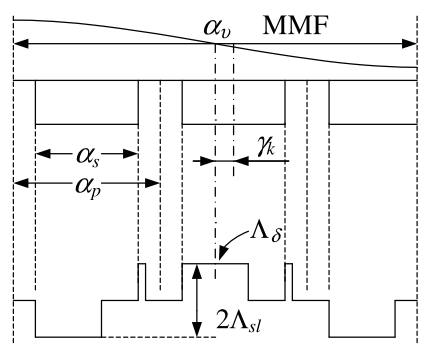

(a)

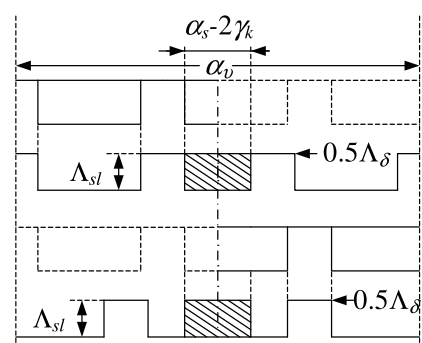

(b)
Fig. 2. Magnetic circuit and average permeance model of $v$-order MMF when $\alpha_{v} \geq \alpha_{s}$. (a) Magnetic circuit and permeance. (b) Equivalent permeance model.

short circuit over the iron pole shoe areas above magnet slots and large reluctance of magnet slots.

In order to simplify the permeance model, we assume the following:

1) the permeance is reduced by $\Lambda_{s l}$ when the flux across one PM slot;

2) the influences of rotor flux barrier bridges are neglected, and hence, the permeance is a square wave;

3) the flux leakages crossing the air gap above flux barrier bridge ribs are ignored;

4) only the unit machine with pole pairs of $p_{0}$ and slot number of $Z_{0}$ is considered in the model.

The permeance of the magnetic circuit that crosses magnet slots, either left or right side, will result in larger reluctance than the short-circuit part. Without loss of generality, the analytical model is discussed in two cases: $\alpha_{v} \geq \alpha_{s}$ and $\alpha_{v}<\alpha_{s}$.

Noticeably, the corresponding permeance of $v$-order harmonic is $v_{0}$-order in the unit machine. However, they are exactly the same, and it will not be distinguished in the derivation.

\section{B. Average Permeance Model When $\alpha_{v} \geq \alpha_{s}$}

For the $\alpha_{v} \geq \alpha_{s}$ case, the relevant permeance model demonstrated in Fig. 2(a) can be simplified by splitting the model into two parts of each $\alpha_{v}$ range using interpole center line, the left part with virtual solid right part and vice versa, as depicted in Fig. 2(b). The whole permeance model can be derived by the superposition of these two parts. For each practical part, the curve shape of permeance is exactly consistent with the airgap permeance excited by fundamental $d$-axis MMF, except the short-circuit shaded part nearby the center line when it crosses with the pole shoe area. The corresponding amplitudes are a half of $d$-axis component, with value of $\left(\Lambda_{\delta} / 2\right)$ when it is above the interpole, and $\left(\Lambda_{\delta} / 2-\Lambda_{s l}\right)$ when it is above the pole shoe. As the virtual parts are considered as solid without magnetic slot, the permeance of these parts can be simply obtained by the symmetric duplication. Examined from Fig. 2(a) and (b), the practical parts without the short-circuit area can be composed together to obtain the average value of $\left(\Lambda_{\delta} / 2-\tau_{s} \Lambda_{s l}\right)$, where $\tau_{s}$ is defined as $\tau_{s}=\alpha_{s} / \alpha_{p}$. As the virtual part is symmetrical to the practical one, the whole average permeance without shaded areas in the figure is $\left(\Lambda_{\delta}-2 \tau_{s} \Lambda_{s l}\right)$.

With regard to the shaded area, there will be $v$ crossings between rotor and interpole center line. The associated variable $\gamma_{k}$ is defined as the angular distance between the $k$ th interpole center of $v$-order MMF and the nearest pole center of the fundamental component, as shown in Fig. 2(a). Assume that $v_{0}=v_{1} t_{1}, p_{0}=p_{1} t_{1}$, where $t_{1}$ is the GCD of $v_{0}$ and $p_{0}$. It can be proved that there will be $v_{1}$ different angular deviation, which can be represented as

$$
\gamma_{k}=k \Delta \gamma+\gamma_{0}, \quad-\frac{\pi}{2 p_{0}} \leq \gamma_{k}<\frac{\pi}{2 p_{0}}
$$

where $k$ is integer, $0 \leq \gamma_{0}<\Delta \gamma$, and $\Delta \gamma$ can be expressed as

$$
\Delta \gamma=\frac{\pi}{v_{1} p_{0}}
$$

Apparently, each $\gamma_{k}$ will repeat $2 t_{0} t_{1}$ times in the machine.

It can be observed from Fig. 2(b) that only the shaded part against the pole shoe will introduce extra magnetic permeance. Consequently, the pole shoe angle $\alpha_{s}$ will affect the air-gap permeance. $\alpha_{s}$ can be expressed in terms of $\Delta \gamma$ and written as

$$
\alpha_{s}=N_{\gamma} \Delta \gamma+\alpha_{r m}, \quad 0<\alpha_{r m}<\Delta \gamma .
$$

If $N_{\gamma}$ is odd, the shaded part can be derived and presented as

$$
\Lambda_{s h}=\left\{\begin{array}{r}
\frac{t_{1}}{\pi}\left(2 N_{\gamma} \alpha_{s}-\left(N_{\gamma}^{2}-1\right) \Delta \gamma-4 \gamma_{0}\right) \Lambda_{s l}, \\
0 \leq \gamma_{0} \leq \frac{\Delta \gamma-\alpha_{r m}}{2} \\
\frac{t_{1}}{\pi}\left(2\left(N_{\gamma}+1\right) \alpha_{s}-\left(N_{\gamma}+1\right)^{2} \Delta \gamma\right) \Lambda_{s l}, \\
\frac{\Delta \gamma-\alpha_{r m}}{2}<\gamma_{0} \leq \frac{\Delta \gamma}{2} .
\end{array}\right.
$$

If $N_{\gamma}$ is even, the shaded part can be represented as

$$
\Lambda_{s h}=\left\{\begin{array}{cc}
\frac{t_{1}}{\pi}\left(2\left(N_{\gamma}+1\right) \alpha_{s}-N_{\gamma}\left(N_{\gamma}+2\right) \Delta \gamma-4 \gamma_{0}\right) \Lambda_{s l}, & 0 \leq \gamma_{0} \leq \frac{\alpha_{r m}}{2} \\
\frac{t_{1}}{\pi}\left(2 N_{\gamma} \alpha_{s}-N_{\gamma}^{2} \Delta \gamma\right) \Lambda_{s l}, & \frac{\alpha_{r m}}{2}<\gamma_{0} \leq \frac{\Delta \gamma}{2}
\end{array}\right.
$$

where $\Lambda_{s h}$ is the permeance component related to the shaded part. It can be noticed that only the case of $\gamma_{0} \leq \Delta \gamma / 2$ is discussed in (7) and (8). For the $\gamma_{0}>\Delta \gamma / 2$ condition, the permeance is simply equal to the case of $\left(\Delta \gamma-\gamma_{0}\right)$ due to the symmetry of the model. The whole average permeance can be proposed as

$$
\Lambda_{v}=\Lambda_{\delta}-2 \tau_{s} \Lambda_{s l}+\Lambda_{s h}
$$

As revealed in the model, the average permeance will be influenced by the smallest degree distance $\gamma_{0}$, which is correlated with the torque angle and spatial order. For the fundamental component $v_{0}=p_{0}$, it can be obtained directly that $v_{1}=1$, and hence, $N_{\gamma}=0, \alpha_{r m}=\alpha_{s}$. Consequently, $\gamma_{0}$ can reach up to the maximum value of $\alpha_{r m} / 2$, which is determined by the initial phase $\theta_{s}$ of MMF. When $\theta_{s}=0$, the corresponding $\gamma_{0}$ is $\alpha_{p} / 2$, and the relative permeance, which is exactly the $d$-axis fundamental component, can be simplified as

$$
\Lambda_{p d}=\Lambda_{\delta}-2 \tau_{s} \Lambda_{s l} .
$$




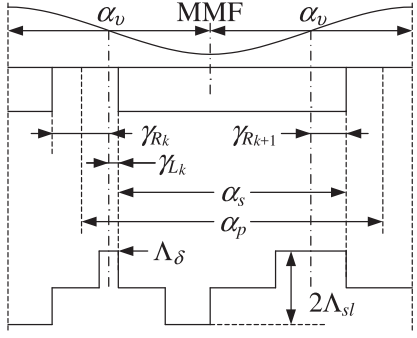

(a)

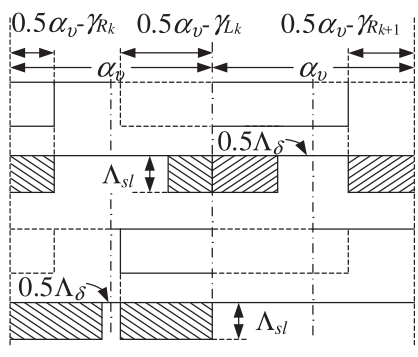

(b)
Fig. 3. Magnetic circuit and average permeance model of $v$-order MMF when $\alpha_{v}<\alpha_{s}$. (a) Magnetic circuit and permeance. (b) Equivalent permeance model.

When $\theta_{s}=\pi / 2$, the corresponding $\gamma_{0}$ is 0 , and the associated $q$-axis permeance can be expressed as

$$
\Lambda_{p q}=\Lambda_{\delta}
$$

The subharmonic component with order of $v_{0}=1$, which is usually one of the most significant magnetic field harmonics in the single-layer FSCW machine, $\gamma_{0}$ also can reach $\alpha_{r m} / 2$. It can be derived similarly that

$$
\Lambda_{\delta}-2 \tau \Lambda_{s l} \leq \Lambda_{1} \leq \Lambda_{\delta}-2 \tau_{s}\left(\frac{p_{0}-1}{p_{0}}\right) \Lambda_{s l}
$$

Usually, it can be simply approximated as $\left(\Lambda_{\delta}-2 \tau_{s} \Lambda_{s l}\right)$. It implies that the magnetic circuit of the $t_{0}$-order MMF is nearly the same as the fundamental $d$-axis component, and the corresponding magnetic field can be reduced in IPMSMs. For other main interharmonics, normally the effects of $\gamma_{0}$ and $\alpha_{r m}$ can be neglected due to a comparatively small value of $\Delta \gamma$. Consequently, the average permeance can be simplified as

$$
\Lambda_{v}=\Lambda_{\delta}-2 \tau_{s} \Lambda_{s l}+\frac{v}{p} \tau_{s}^{2} \Lambda_{s l}
$$

\section{Average Permeance Model When $\alpha_{v}<\alpha_{s}$}

For $\alpha_{v}<\alpha_{s}$, the permeance model can be similarly separated into two parts by distinguishing the magnet slot into two parts based on the relative position to the rotor. As shown in Fig. 3(b), the left-side part of the magnetic slot always goes with the right part even it allocates on the right side of interpole center line. There must be $2 p$ crossing $v$-order poles for both left- and rightside magnetic slots, of which the corresponding permeance will be reduced by the slot portion as shadow marked in Fig. 3(b), while for the poles without the crossing area, the magnetic circuit will be in short circuit by the rotor shoes or interpole sectors and, hence, maintain as a constant of $\Lambda_{\delta} / 2$ for both separated parts. Consequently, the whole permeance can be derived by the subtraction of the constant part $\Lambda_{\delta}$ and the crossing shaded part. As a resemblance, the angle deviation can be written as

$$
\left\{\begin{aligned}
\gamma_{L_{k}}=k \Delta \gamma_{L}+\gamma_{L_{0}}, & -\frac{\pi}{2 v_{0}} \leq \gamma_{L_{k}}<\frac{\pi}{2 v_{0}} \\
\gamma_{R_{k}}=k \Delta \gamma_{R}+\gamma_{R_{0}}, & -\frac{\pi}{2 v_{0}} \leq \gamma_{R_{k}}<\frac{\pi}{2 v_{0}}
\end{aligned}\right.
$$

where $0 \leq \gamma_{L_{0}}<\Delta \gamma_{L}$, and $0 \leq \gamma_{R_{0}}<\Delta \gamma_{R} \cdot \Delta \gamma_{L}$ and $\Delta \gamma_{R}$ are given by

$$
\Delta \gamma_{L}=\Delta \gamma_{R}=\frac{\pi}{p_{1} v_{0}}=\Delta \gamma
$$

Obviously, there are $p_{1}$ different degree distance for both $\gamma_{L_{k}}$ and $\gamma_{R_{k}}$. As a resemblance to models (7) and (8), the permeance of the shaded part can be derived. When $p_{1}$ is odd, the shaded part can be presented as

$$
\Lambda_{s h}=\frac{p}{v} \Lambda_{s l}+\frac{t_{1}}{\pi}\left(\Delta \gamma-2 \gamma_{L_{0}}-2 \gamma_{R_{0}}\right) \Lambda_{s l} .
$$

When $p_{1}$ is even, it can be derived as

$$
\Lambda_{s h}=\frac{p}{v} \Lambda_{s l} .
$$

As a consequence, the whole average permeance can be expressed as

$$
\Lambda_{v}=\Lambda_{\delta}-\Lambda_{s h} .
$$

Obviously, when $v_{0} \gg p_{0}$, the average permeance can be approximated as $\Lambda_{\delta}$. It means that for high-order harmonics, the corresponding magnetic circuit is close to the fundamental $q$-axis component.

The lower order slot harmonic components are normally the major harmonic components in the magnetic field, and $\Delta \gamma$ is very small as $p_{0}$ and $v_{0}$ are coprime in most of the FSCW configuration. Hence, the corresponding average permeance (18) can also be approximately rewritten as

$$
\Lambda_{v}=\Lambda_{\delta}-\frac{p_{0}}{v_{0}} \Lambda_{s l}
$$

\section{HARMONiC MAGNETIC FIELD RATIO AND SIMULATION VALIDATION}

\section{A. Fundamental Spatial Air-Gap Magnetic Field Components}

As proposed in [22], the fundamental spatial air-gap magnetic field component can be presented as

$$
b_{0}(\theta, t)=B_{0} \cos \left(p \theta-\omega_{1} t-\delta\right)
$$

where

$$
B_{0}=\frac{\sqrt{\left(L_{a d} i_{d}+\psi_{f}\right)^{2}+\left(L_{a q} i_{q}\right)^{2}}}{\alpha \tau_{p} N K_{w p} L_{\mathrm{ef}}}
$$

where the inductances are the ones contributed only by the fundamental spatial component and can be approximately expressed by neglecting the contributions of high-order rotor saliency components as

$$
L_{a d}=L_{p}-L_{s l}, \quad L_{a q}=L_{p}+L_{s l} .
$$

$L_{p}$ is related to the mean value of permeance for the fundamental magnetic field, and $L_{s l}$ is the part introduced by the saliency component $\Lambda_{s l}$

Meanwhile, the corresponding component contributed by armature reaction can be directly decoupled from the overall one and expressed as

$$
b_{a}^{p}(\theta, t)=B_{a}^{p} \cos \left(p \theta-\omega_{1} t-\theta_{a}\right)
$$


where

$$
B_{a}^{p}=\frac{\sqrt{L_{a d}^{2} i_{d}^{2}+L_{a q}^{2} i_{q}^{2}}}{\alpha \tau_{p} N K_{w p} L_{\mathrm{ef}}}, \quad \theta_{a}=\pi+\arctan \frac{L_{a q} i_{q}}{L_{a d} i_{d}} .
$$

\section{B. Magnetic Field Interharmonic Components}

As certain spatial interharmonic components of the air-gap magnetic field from armature reaction can be significant in IPMSM with FSCW configuration, it is essential to include them in the investigation especially under heavy-load conditions. As slot interharmonic permeance can be approximately considered as constant, the corresponding flux density can be simplified as

$$
B_{a}^{v} \approx F_{v} \Lambda_{v} .
$$

Meanwhile, a virtual nonsalient flux density is defined as

$$
B_{a} \approx F_{p} \Lambda_{p}
$$

where $\Lambda_{p}=\Lambda_{\delta}-\tau_{s} \Lambda_{s l}$. The corresponding inductance of $\Lambda_{p}$ is $L_{p}$. By substituting (3) into (25) and (26), the amplitude of the $v$-order spatial component can be obtained as

$$
B_{a}^{v}=K_{v} B_{a}, \quad K_{v}=\frac{p K_{w v} \Lambda_{v}}{|v| K_{w p} \Lambda_{p}} .
$$

Therefore, the $v$-order spatial component of the armature reaction air-gap magnetic field can be expressed as

$$
b_{a}^{v}(\theta, t)=K_{v} B_{a} \cos \left(v \theta-\omega_{1} t-\theta_{s}\right)
$$

where $B_{a}$ is the amplitude of the fundamental spatial components and can be easily derived from (24) by neglecting the salient part $L_{s l}$ in the inductances as

$$
B_{a}=\frac{L_{p} i_{s}}{\alpha \tau_{p} N K_{w p} L_{\mathrm{ef}}} .
$$

Based on the proposed model, the ratio of $\Lambda_{v}$ and $\Lambda_{p}$ can be calculated from the proposed permeance model, which is also associated with inductance parameters $L_{a d}$ and $L_{a q}$. Moreover, the spatial order $v$ involved in (27) and (28) can be negative. That is because the rotational information included in the frequency component in (2) is moved to spatial order part.

\section{Validation of Interharmonics in FSCW PMSMs}

As discussed in the previous section, the influence of the rotor position can be neglected for the main average permeance harmonics. Consequently, the influence of rotor saliency on slot magnetic field components, which are the main interharmonics in FSCW PMSMs, can be ignored. Fig. 4 demonstrates the simulation results of armature reaction obtained from different pole-slot configurations of double-layer FSCW IPMSMs, with $d q$ fundamental components in Fig. 4(a). It can be seen from the figure that significant deviation between $d$ - and $q$-axes magnetic field components exists due to the rotor saliency.

As one of the most significant interharmonics, the $(Z-p)$ order component is investigated as well. The corresponding $d$ - and $q$-axis components of $(Z-p)$-order magnetic field are also demonstrated in Fig. 4(b). As expected, the $(Z-p)$-order components are very close to each other for all configurations, due to the trivial influence from the rotor saliency.

Furthermore, the comparison between analytical results and FEM results of the magnetic field ratio of $(Z-p)$-order interharmonic is also carried out and depicted in Fig. 4(c). Close agreement has been achieved for all configurations, and hence, the validity and accuracy of magnetic harmonic model have been sufficiently underpinned. Specifically, for the cases of $2 p<Z$, $K_{Z-p}$-order harmonics can be further approximated as $p /|v|$ for quick assessment of $(Z-p)$-order harmonic.

In the single-layer winding machine, the $t_{0}$-order one is another significant harmonic, and the magnetic field ratio model (27) can still be employed for the harmonic prediction. However, it might result in slightly larger than the practical value when $t_{0} \ll p$, due to the evident flux leakages in the stator teeth between interharmonic MMF pole pairs.

\section{Analytical Model of Sideband HARMONICS OF SPWM}

\section{A. Analytical Sideband Current Harmonics}

The SPWM voltage-source inverter (VSI) drive will generate a cluster of sideband voltage and current harmonics about integer multiples of the switching frequency in the stator windings of the PMSM. In the rotor reference frame, the $\left(\omega_{s} \pm 3 \omega_{1}\right)$ order harmonic components are the main ones in the first carrier frequency domain, while the $\left(2 \omega_{s}\right)$-order ones are the main components in second carrier frequency. As a resemblance to the analytical sideband current model in SVPWM [23], the corresponding component can be similarly derived as

$$
\begin{aligned}
& \left\{\begin{array}{l}
i_{d_{-13}}=-\frac{C_{12} U_{\mathrm{dc}} \sin \left(\omega_{s} t \pm 3 \omega_{1} t \mp \delta\right)}{2\left(\omega_{s} \pm 3 \omega_{1}\right) L_{d}} \\
i_{q_{-13}}=\mp \frac{C_{12} U_{\mathrm{dc}} \cos \left(\omega_{s} t \pm 3 \omega_{1} t \mp \delta\right)}{2\left(\omega_{s} \pm 3 \omega_{1}\right) L_{q}}
\end{array}\right. \\
& \left\{\begin{array}{l}
i_{d_{-20}}=-\frac{C_{21} U_{\mathrm{dc}} \sin \delta \sin \left(2 \omega_{s} t\right)}{2 \omega_{s} L_{d}} \\
i_{q \_20}=\frac{C_{21} U_{\mathrm{dc}} \cos \delta \sin \left(2 \omega_{s} t\right)}{2 \omega_{s} L_{q}}
\end{array}\right.
\end{aligned}
$$

where $C_{12}$ and $C_{21}$ can be approximated as

$$
C_{12} \approx \frac{4}{\pi} J_{2}\left(\frac{M \pi}{2}\right), \quad C_{21} \approx-\frac{2}{\pi} J_{1}(M \pi)
$$

where $M \in[0,1]$. It should be noted in (30) and (31) that $L_{d}$ and $L_{q}$ are the whole inductances of winding, including all of flux leakage and the harmonic MMF components. By applying the inverse Park transformation, the $\left(\omega_{s} \pm 3 \omega_{1}\right)$-order current harmonics can be turned into $\left(\omega_{s} \pm 2 \omega_{1}\right)$ and $\left(\omega_{s} \pm 4 \omega_{1}\right)$-order phase current harmonics in the stator stationary frame, while the $\left(2 \omega_{s}\right)$-order one can be transformed into $\left(2 \omega_{s} \pm \omega_{1}\right)$-order harmonics. The respective amplitudes of those harmonics in the 


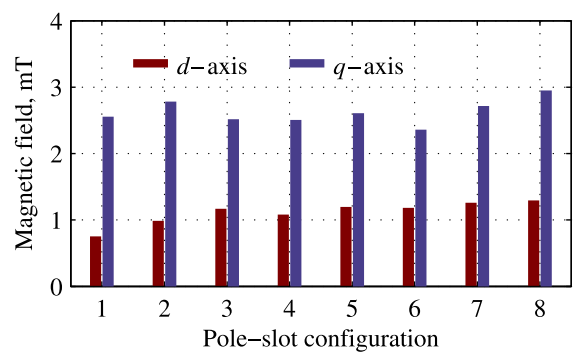

(a)

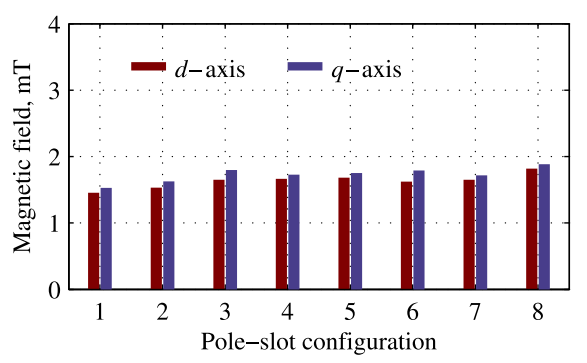

(b)

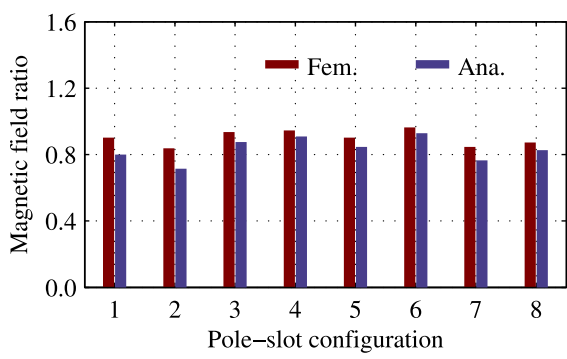

(c)

Fig. 4. Simulation validation of interharmonics. (a) Fundamental $d$ - and $q$-components. (b) $(Z-p)$-order $d$ - and $q$-harmonics. (c) $K_{Z}-p$. (1: 8-pole 9-slot. 2: 10-pole 12-slot. 3: 14-pole 15-slot. 4: 20-pole 21-slot. 5: 22-pole 24-slot. 6: 26-pole 27-slot. 7: 26-pole 30-slot. 8: 38-pole 42-slot).

stator stationary frame can be obtained as

$$
\begin{aligned}
& \left\{\begin{array}{l}
i_{s_{-} 12}=\frac{U_{\mathrm{dc}} C_{12}}{4\left(\omega_{s} \pm 3 \omega_{1}\right)}\left(\frac{1}{L_{d}}+\frac{1}{L_{q}}\right) \\
i_{s_{-} 14}=\frac{U_{\mathrm{dc}} C_{12}}{4\left(\omega_{s} \pm 3 \omega_{1}\right)}\left(\frac{1}{L_{d}}-\frac{1}{L_{q}}\right)
\end{array}\right. \\
& i_{s_{-} 21}=\frac{U_{\mathrm{dc}} C_{21}}{4 \omega_{s}} \sqrt{\frac{\sin ^{2} \delta}{L_{d}^{2}}+\frac{\cos ^{2} \delta}{L_{q}^{2}}} .
\end{aligned}
$$

Besides those first and second sideband harmonic components, there are usually more sideband components, such as $\left(2 \omega_{s} \pm 5 \omega_{1}\right),\left(2 \omega_{s} \pm 7 \omega_{1}\right)$-order ones, which are normally much smaller in most of the cases and hence ignored for further discussion in this paper. However, the analytical derivations of those sideband harmonics can be carried out with the same approach. One of the implications from (33) is that the $\left(\omega_{s} \pm 4 \omega_{1}\right)$ order sideband current harmonics in the PMSM drive with the SPWM technique originate from the difference between the $d$-axis and $q$-axis inductances (saliency). Therefore, they almost do not exist in the machine with small saliency such as surfacemounted PMSMs but have nearly the same amplitudes as the $\left(\omega_{s} \pm 2 \omega_{1}\right)$-order ones in the machine with large saliency.

\section{B. Analytical Sideband Air-Gap Flux Density}

With the same procedure of the sideband magnetic field in SVPWM [22], the corresponding components in SPWM can be presented as

$$
\left\{\begin{array}{l}
b_{12}^{p}= \pm B_{12}^{p} \cos \left(p \theta \pm\left(\omega_{s} \pm 2 \omega_{1}\right) t-\theta_{12}\right) \\
b_{14}^{p}= \pm B_{14}^{p} \cos \left(p \theta \mp\left(\omega_{s} \pm 4 \omega_{1}\right) t-\theta_{14}\right)
\end{array}\right.
$$

The amplitudes and initial phase are given by

$$
\left\{\begin{array}{l}
B_{12}^{p}=\frac{\sigma_{1} C_{12} U_{\mathrm{dc}}}{4 \alpha \tau_{p} N K_{d p} L_{\mathrm{ef}}\left(\omega_{s} \pm 3 \omega_{1}\right)} \\
B_{14}^{p}=\frac{\sigma_{2} C_{12} U_{\mathrm{dc}}}{4 \alpha \tau_{p} N K_{d p} L_{\mathrm{ef}}\left(\omega_{s} \pm 3 \omega_{1}\right)}
\end{array}\right.
$$

and

$$
\theta_{12}=\frac{3 \pi}{2}+\delta, \quad \theta_{14}=\frac{\pi}{2}-\delta
$$

where

$$
\sigma_{1}=L_{a d} / L_{d}+L_{a q} / L_{q}, \quad \sigma_{2}=L_{a q} / L_{q}-L_{a d} / L_{d} .
$$

As a resemblance, the resultant sideband harmonic magnetic field of $\left(2 \omega_{s}\right)$-order current harmonics can be derived as

$$
b_{21}^{p}= \pm B_{21}^{p} \cos \left(p \theta \mp\left(2 \omega_{s} \pm \omega_{1}\right) t-\theta_{21}^{p}\right)
$$

where

$$
\begin{aligned}
B_{21}^{p} & =\frac{\sigma_{3} C_{21} U_{\mathrm{dc}}}{4 \alpha \tau N K_{d p} L_{\mathrm{ef}} \omega_{s}}, \quad \theta_{21}^{p}=\arctan \frac{L_{q} L_{a d} \sin \delta}{L_{d} L_{a q} \cos \delta} \\
\sigma_{3} & =\sqrt{\left(\frac{L_{a d}}{L_{d}} \sin \delta\right)^{2}+\left(\frac{L_{a q}}{L_{q}} \cos \delta\right)^{2}} .
\end{aligned}
$$

Moreover, the derivations of the $v$-order spatial interharmonic components of the air-gap magnetic field generated by the corresponding sideband current harmonic components can follow exactly the same approach as the ones of the fundamental current component. Thus, the respective $v$-order spatial components in the stator stationary reference frame can be presented as

$$
\left\{\begin{array}{l}
b_{12}^{v}= \pm K_{v} B_{12} \cos \left(v \theta \pm\left(\omega_{s} \pm 2 \omega_{1}\right) t-\theta_{12}^{h}\right) \\
b_{14}^{v}= \pm K_{v} B_{14} \cos \left(v \theta \mp\left(\omega_{s} \pm 4 \omega_{1}\right) t-\theta_{14}^{h}\right) \\
b_{21}^{v}= \pm K_{v} B_{21} \cos \left(v \theta \mp\left(2 \omega_{s} \pm \omega_{1}\right) t-\theta_{21}^{h}\right)
\end{array}\right.
$$

where

$$
\left\{\begin{aligned}
B_{12} & =\frac{\sigma_{4} C_{12} U_{\mathrm{dc}}}{4 \alpha \tau_{p} N K_{d p} L_{\mathrm{ef}}\left(\omega_{s} \pm 3 \omega_{1}\right)} \\
B_{14} & =\frac{\sigma_{5} C_{12} U_{\mathrm{dc}}}{4 \alpha \tau_{p} N K_{d p} L_{\mathrm{ef}}\left(\omega_{s} \pm 3 \omega_{1}\right)} \\
B_{21} & =\frac{\sigma_{6} C_{21} U_{\mathrm{dc}}}{4 \alpha \tau_{p} N K_{d p} L_{\mathrm{ef}} \omega_{s}}
\end{aligned}\right.
$$

and

$$
\theta_{21}^{h}=\arctan \left(L_{q} \sin \delta / L_{d} \cos \delta\right)
$$

where

$$
\left\{\begin{array}{l}
\sigma_{4}=L_{p} / L_{q}+L_{p} / L_{d}, \sigma_{5}=L_{p} / L_{d}-L_{p} / L_{q} \\
\sigma_{6}=\sqrt{\left(\frac{L_{p}}{L_{d}} \sin \delta\right)^{2}+\left(\frac{L_{p}}{L_{q}} \cos \delta\right)^{2}} .
\end{array}\right.
$$

\section{Sideband Radial Force Density in IPMSMs}

The sideband magnetic field will interact with the PM and fundamental current magnetic field components to produce highfrequency radial force, which will acting on the stator system to 


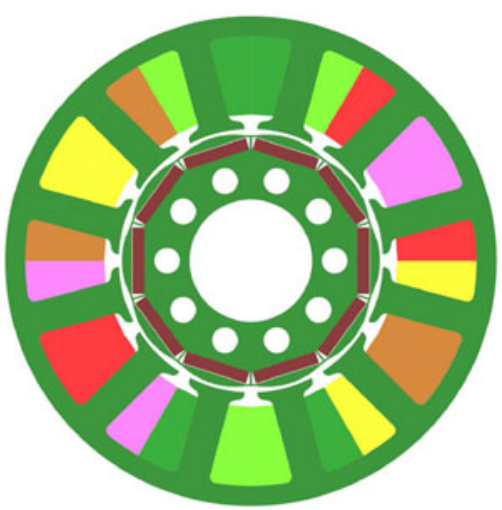

Fig. 5. Cross section of the experimental IPMSM.

produce vibration and acoustic noise. According to the Maxwell stress tensor theory, by neglecting the insignificant component, the sideband radial force density can be approximated as

$$
p_{r}(\theta, t) \approx-\frac{\left(b_{0}(\theta, t)+b_{a}^{v}(\theta, t)\right) b_{\mu}^{v}(\theta, t)}{\mu_{0}} .
$$

By substituting the fundamental components models (20), (28) and sideband ones (35), (39), (41) into (45), the sideband radial force density can be calculated. It can be noticed that $v \neq p$ for $b_{a}^{v}$, as the fundamental component has been included in $b_{0}$. Although the armature reaction flux linkage can be close to the PM flux linkage when the machine running under the heavyload condition, the associated interharmonic magnetic field can be ignored in most of the cases. The armature reaction linkage is composed of several interharmonics of similar values, and hence, each part is normally much smaller than the fundamental flux density. Meanwhile, the damping factor takes more important role for high-frequency resonance, and hence, the resonance will not be as significant as the low-frequency cases. As a consequence, normally only the large component of sideband radial force is of interest, and (45) can be further approximated as

$$
p_{r}(\theta, t) \approx-\frac{b_{0}(\theta, t) b_{\mu}^{v}(\theta, t)}{\mu_{0}} .
$$

Based on the proposed radial force model, the sideband vibration of SPWM can be derived by the analytical vibration model [22], [25]. In order to improve the prediction accuracy, the mechanical finite-element analysis (FEA) is employed for the stator natural frequency calculation.

\section{EXPERIMENTAL VALIDATION}

\section{A. Experimental Drive System and Sideband Current Validation}

In order to validate the analytical derivations provided by the foregoing sections, comprehensive experimental tests are carried out. The IPMSM, whose cross section is shown in Fig. 5, is driven by a two-level VSI with the SPWM technique and $i_{d}=0$ control strategy, and the main parameters of the machine are given in Table I. The PMSM drive is conducted under the no-load condition, and thus, the interference comes from the mechanical connection and load can be avoided. In fact,
TABLE I

Key Design Parameters of the Machine

\begin{tabular}{lccc}
\hline \hline Machine Parameter & Value & Machine Parameter & Value \\
\hline Pole number & 10 & Slot number & 12 \\
Shell diameter & $98 \mathrm{~mm}$ & Shell height & $115 \mathrm{~mm}$ \\
Stator outer diameter & $76 \mathrm{~mm}$ & Axial stator length & $70 \mathrm{~mm}$ \\
Stator inner diameter & $41 \mathrm{~mm}$ & Stator yoke height & $3 \mathrm{~mm}$ \\
DC-ink voltage & $42 \mathrm{~V}$ & PWM frequency & $4.5 \mathrm{kHz}$ \\
Rated speed & $300 \mathrm{r} / \mathrm{min}$ & Maximum speed & $750 \mathrm{r} / \mathrm{min}$ \\
\hline \hline
\end{tabular}

the sideband currents and associated vibration can be investigated by different speed conditions, as the modulation index is the main parameter determining the sideband harmonics.

The experiment tests on the proposed PMSM are first carried out at an operational speed of $600 \mathrm{r} / \mathrm{min}$. The experimental phase current spectrum is captured and depicted in Fig. 6. It can be directly observed from Fig. 6(b) and (c) that the main sideband current harmonic components in first and second carrier frequency domains are the $\left(\omega_{s} \pm 2 \omega_{1}\right)$, and $\left(2 \omega_{s} \pm \omega_{1}\right)$-order ones. Different from SVPWM, the $\left(\omega_{s} \pm 4 \omega_{1}\right)$ components are rather small due to close $d$ - and $q$-axis inductance components. Consequently, these harmonics will not be taken into consideration in further discussion. Moreover, the others such as $\left(2 \omega_{s} \pm 5 \omega_{1}\right)$ and $\left(2 \omega_{s} \pm 7 \omega_{1}\right)$-order components are too small to be considered.

In order to experimentally validate the analytical models quantitatively, the amplitude variations of the main sideband current harmonics with different modulation ratios are further examined for the proposed PMSM. The experimental tests of the prototype IPMSM with different speeds from 60 to $720 \mathrm{r} / \mathrm{min}$ under the no-load condition are conducted to achieve various modulation ratios. The resultant amplitudes of the main sideband current harmonic components, including $\left(\omega_{s} \pm 2 \omega_{1}\right)$-, and $\left(2 \omega_{s} \pm \omega_{1}\right)$-order ones, are compiled from the experimental results and compared with the analytical ones in Fig. 7(a) and (b), respectively. The figures demonstrate very close agreements between the analytical and experimental results for the harmonic components and validate that the proposed analytical models can deliver reasonably accurate predictions for the SPWM scheme.

\section{B. Natural Frequency and Sideband Radial Force Density}

According to model (46), there will be an abundance of sideband spatial order radial force components in the machine, such as 0 -order, $2 p$-order, $(Z-2 p)$-order, $\left(p-t_{0}\right)$-order, and $\left(p+t_{0}\right)$-order items. By substituting the associated flux density models into the radial force model, the main components in first and second sidebands are demonstrated in Table II.

The corresponding natural frequencies of these items become essential for the vibration prediction, and the structural FEA is employed to calculate the eigenvalue. The low-order natural frequencies of the stator system, including 0 -order, $(Z-2 p)=$ 2-order, and $\left(p-t_{0}\right)=4$-order components, are obtained and depicted in Fig. 8. Despite its low spatial order value, the natural frequency of 0 -order is much higher than 2- and 4-order components, and hence, the corresponding vibration items can 


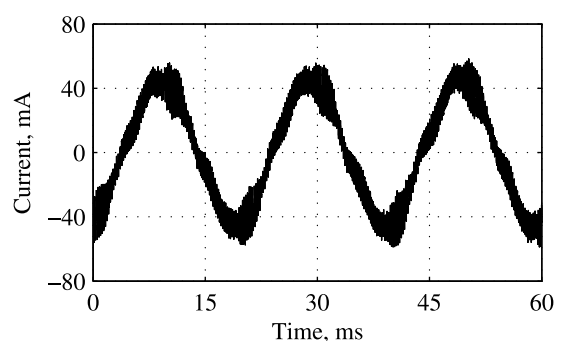

(a)

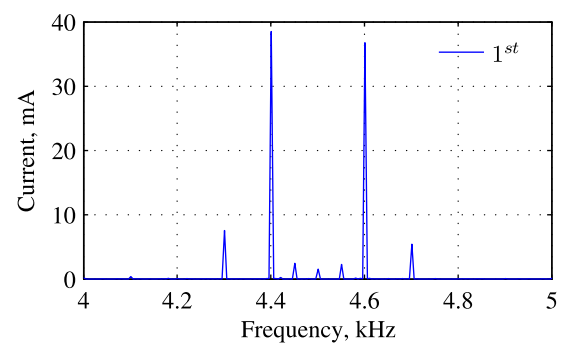

(b)

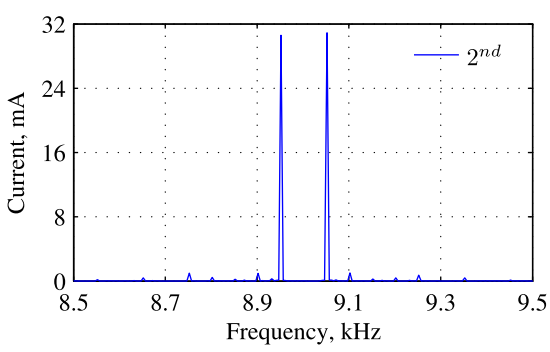

(c)

Fig. 6. Phase current spectrum at a rotational speed of 600r/min. (a) Current waveform. (b) First sideband components. (c) Second sideband components.

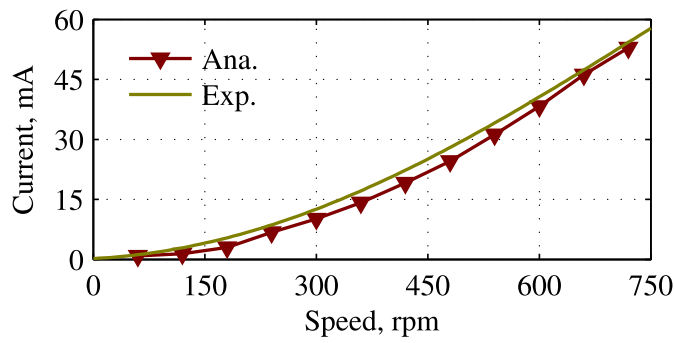

(a)

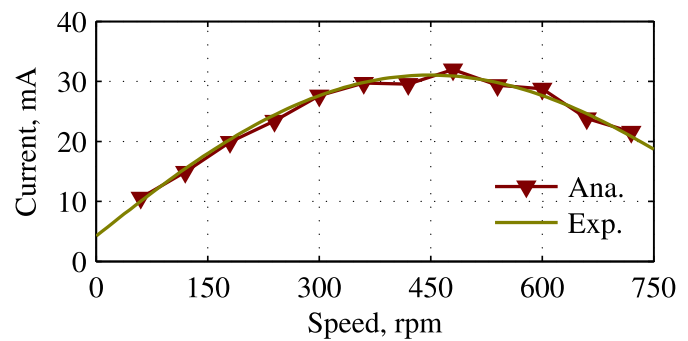

(b)

Fig. 7. Sideband current harmonics under different speed conditions. (a) $\omega_{s} \pm 2 \omega_{1}$. (b) $2 \omega_{s} \pm \omega_{1}$.
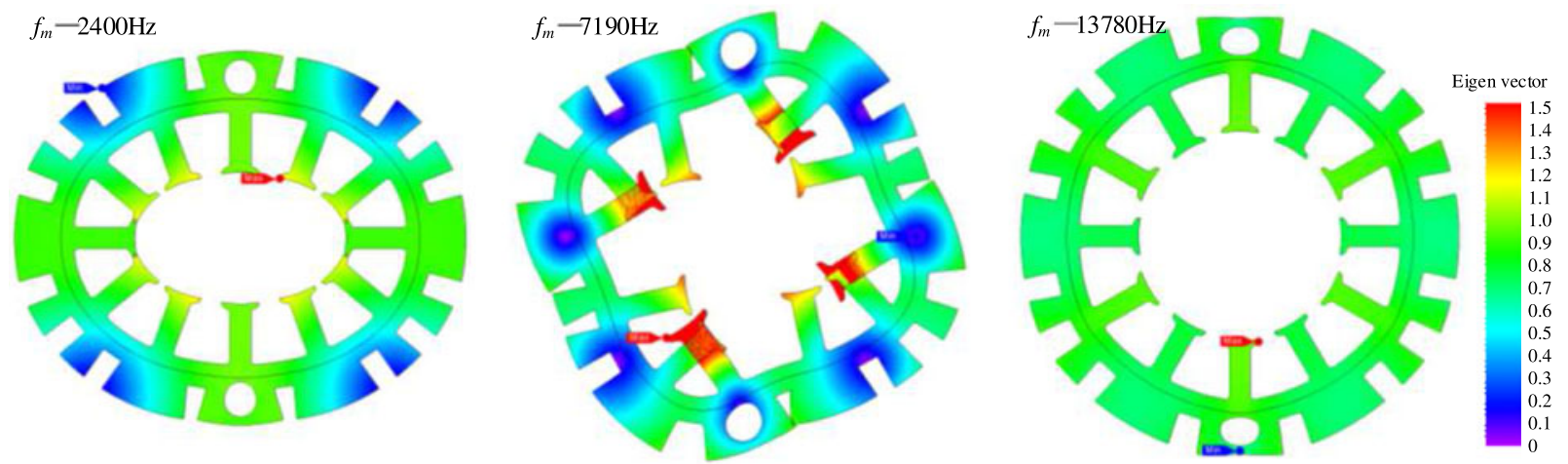

Fig. 8. Stator system natural frequency analysis. (a) Second order. (b) Fourth order. (c) Zeroth order.

TABLE II

MAIN SidEBAND RADIAL FORCE DENSITY COMPONENTS

\begin{tabular}{lccc}
\hline \hline Order & Frequency & Phase & Amplitude \\
\hline 0 & $\mp\left(\omega_{s} \pm 3 \omega_{1}\right)$ & $\theta_{12}-\delta$ & $B_{0} B_{12}^{p} /\left(2 \mu_{0}\right)$ \\
0 & $\pm 2 \omega_{s}$ & $\theta_{21}^{p}-\delta$ & $B_{0} B_{21}^{p} /\left(2 \mu_{0}\right)$ \\
$Z-2 p$ & $\pm\left(\omega_{s} \pm \omega_{1}\right)$ & $-\theta_{12}-\delta$ & $K_{Z}-p B_{0} B_{12} /\left(2 \mu_{0}\right)$ \\
$Z-2 p$ & $\mp\left(2 \omega_{s} \pm 2 \omega_{1}\right)$ & $-\theta_{21}^{h}-\delta$ & $K_{Z-p} B_{0} B_{21} /\left(2 \mu_{0}\right)$ \\
$p-t_{0}$ & $\mp\left(\omega_{s} \pm \omega_{1}\right)$ & $\delta+\theta_{12}$ & $K_{t_{0}} B_{0} B_{12} /\left(2 \mu_{0}\right)$ \\
$p-t_{0}$ & $\pm\left(2 \omega_{s} \pm 2 \omega_{1}\right)$ & $\delta+\theta_{21}^{h}$ & $K_{t_{0}} B_{0} B_{21} /\left(2 \mu_{0}\right)$ \\
$p+t_{0}$ & $\pm\left(\omega_{s} \pm 3 \omega_{1}\right)$ & $\delta-\theta_{12}$ & $K_{t_{0}} B_{0} B_{12} /\left(2 \mu_{0}\right)$ \\
$p+t_{0}$ & $\mp 2 \omega_{s}$ & $\delta-\theta_{21}^{h}$ & $K_{t_{0}} B_{0} B_{21} /\left(2 \mu_{0}\right)$ \\
$2 p$ & $\mp\left(\omega_{s} \pm \omega_{1}\right)$ & $\theta_{12}+\delta$ & $B_{0} B_{12}^{p} /\left(2 \mu_{0}\right)$ \\
$2 p$ & $\pm\left(2 \omega_{s} \pm 2 \omega_{1}\right)$ & $\theta_{21}^{p}+\delta$ & $B_{0} B_{21}^{p} /\left(2 \mu_{0}\right)$ \\
\hline \hline
\end{tabular}

be ignored. For the higher order components, the eigenvalue is rather bigger than the low-order components and far away from the switching requency involved for investigation. Consequently, they can be neglected as well.
As aforementioned, $(Z-2 p)=2$-order and $\left(p-t_{0}\right)=$ 4-order radial forces are further investigated for the sideband vibration. The corresponding force density of the machine at different speeds has been given in Fig. 9. Apparently, the $\left(p-t_{0}\right)$ order sideband components are comparatively small due to its smaller magnetic field coefficient. Furthermore, the characteristics of the sideband force density components are basically comport well with associated sideband current. The $\left(\omega_{s} \pm \omega_{1}\right)$ order components will gradually increase as the speed increases, while the amplitudes of the $\left(2 \omega_{s} \pm 2 \omega_{1}\right)$-order ones will first increase to reach their peaks and then start to decrease as the speed increases.

\section{Experimental Validation of Sideband Vibration}

The stator vibrations of the PMSM under $600 \mathrm{r} / \mathrm{min}$ with the no-load condition are measured and illustrated in Fig. 10 as well. The measure point of the vibration is located in the center of the machine case surface along the axial direction. It can be easily 


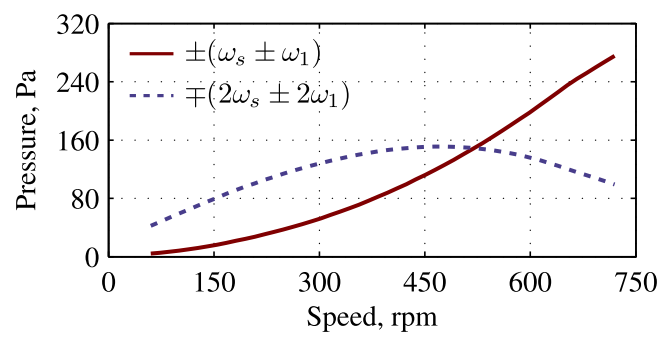

(a)

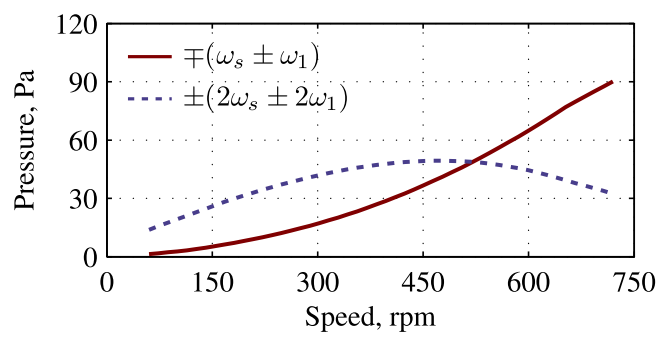

(b)

Fig. 9. Radial force density at different speed condition: (a) $(Z-2 p)$-order; (b) $\left(p-t_{0}\right)$-order.

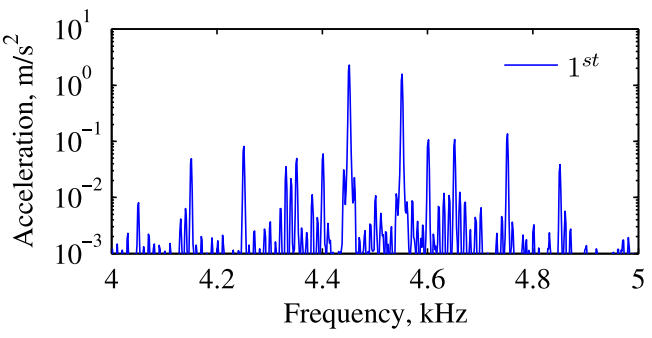

(a)

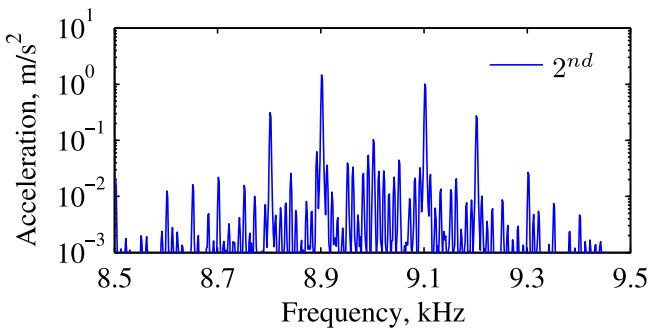

(b)

Fig. 10. Machine vibration spectrum at a rotational speed of 600r/min. (a) First sideband components. (b) Second sideband components.

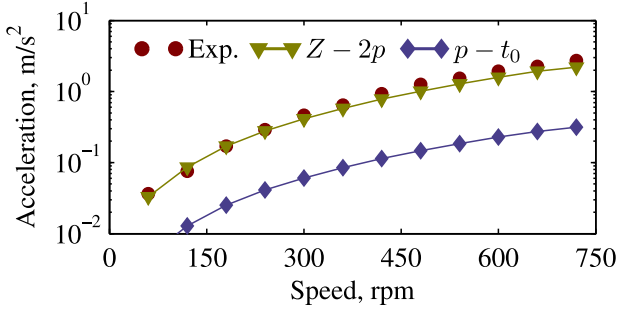

(a)

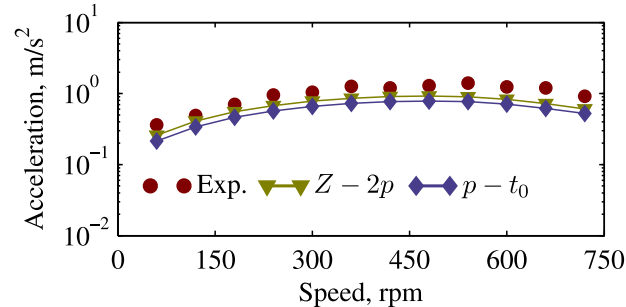

(b)

Fig. 11. Machine vibration experimental validation at different speeds. (a) $\left(\omega_{s} \pm \omega_{1}\right)$-order. (c) $\left(2 \omega_{s} \pm 2 \omega_{1}\right)$-order.

observed that $\left(\omega_{s} \pm \omega_{1}\right)$ and $\left(2 \omega_{s} \pm 2 \omega_{1}\right)$-order components, which are $4450 \mathrm{~Hz} / 4550 \mathrm{~Hz}$ and $8900 \mathrm{~Hz} / 9100 \mathrm{~Hz}$, respectively, are the main sideband machine vibration components in first and second carrier frequency domains. There are also components such as $\left(\omega_{s} \pm 5 \omega_{1}\right)$-, and $\left(2 \omega_{s} \pm 4 \omega_{1}\right)$-order ones, which are caused by $\left(\omega_{s} \pm 4 \omega_{1}\right)$ - and $\left(2 \omega_{s} \pm 5 \omega_{1}\right)$-order sideband currents. They are usually very trivial and can be neglected. Insignificant $\left(\omega_{s} \pm 3 \omega_{1}\right)$-order components, which are introduced by 0 -order radial force, can be also identified in the figure. However, the acceleration amplitude of these components is rather small as the large eigenvalue of the stator system.

Moreover, the amplitude variations of the main vibration frequency with different speeds are further examined and compared for both analytical and experimental results. The corresponding amplitudes of the main vibration frequency components from analytical models, and experimental tests, are derived and compared in Fig. 11. Both $(Z-2 p)$-order and $\left(p-t_{0}\right)$-order components are depicted in the figure. The $(Z-2 p)$-orders are the predominant components in the first sideband, while $\left(p-t_{0}\right)$-order components also become one of the major components in the second sideband as associated frequency is close to the eigenvalue, and hence, resonance will be excited. It can be found that satisfactory agreements between the analytical model and experimental results for all of these sideband orders have been successfully demonstrated, and it is reasonable to conclude that the validation of the proposed model is sufficiently approved.

\section{CONCLUSION}

This paper focuses on the analytical investigation of sideband vibration in the FSCW IPMSM drive with the SPWM technique. A new model of interharmonic in FSCW IPMSMs is analytically developed. Novel analytical sideband current and associated radial force density models are derived accordingly. FEA simulation and comprehensive experiments are carried out to underpin the proposed analytical models. Close agreements between analytical and experimental results have been achieved for both sideband current and vibration components, which demonstrate that the proposed analytical approaches can deliver fast and accurate prediction for FSCW IPMSM drives. Most importantly, the validated models can offer detailed and insightful revelations of various factors that affect the corresponding sideband vibration components. 


\section{REFERENCES}

[1] A. M. EL-Refaie, "Fractional-slot concentrated-windings synchronous permanent magnet machines: Opportunities and challenges," IEEE Trans. Ind. Electron., vol. 57, no. 1, pp. 107-121, Jan. 2010.

[2] D. Gerling, "Analysis of the magnetomotive force of a three-phase winding with concentrated coils and different symmetry features," in Proc. Int. Conf. Elect. Mach. Syst., Oct. 2008, pp. 2832-2837.

[3] M. V. Cistelecan, F. J. T. E. Ferreira, and M. Popescu, "Three phase tooth-concentrated multiple-layer fractional windings with low space harmonic content," in Proc. IEEE Energy Convers. Congr. Expo., Sep. 2010, pp. 1399-1405.

[4] G. Dajaku and D. Gerling, "A novel 24-slots/10-poles winding topology for electric machines," in Proc. IEEE Int. Elect. Mach. Drives Conf., May 2011, pp. 65-70.

[5] G. Dajaku and D. Gerling, "A novel 12-teeth/10-poles pm machine with flux barriers in stator yoke," in Proc. XXth Int. Conf. Elect. Mach., Sep. 2012, pp. 36-40.

[6] G. Dajaku, W. Xie, and D. Gerling, "Reduction of low space harmonics for the fractional slot concentrated windings using a novel stator design," IEEE Trans. Magn., vol. 50, no. 5, pp. 1-12, May 2014.

[7] G. Dajaku and D. Gerling, "Air-gap flux density characteristics of salient pole synchronous permanent-magnet machines," IEEE Trans. Magn., vol. 48, no. 7, pp. 2196-2204, Jul. 2012.

[8] A. K. Putri, S. Rick, D. Franck, and K. Hameyer, "Application of sinusoidal field pole in a permanent-magnet synchronous machine to improve the NVH behavior considering the MTPA and MTPV operation area," IEEE Trans. Ind. Appl., vol. 52, no. 3, pp. 2280-2288, May 2016.

[9] Y. Asano, Y. Honda, H. Murakami, Y. Takeda, and S. Morimoto, "Novel noise improvement technique for a PMSM with concentrated winding," in Proc. Power Convers. Conf., 2002, vol. 2, pp. 460-465.

[10] P. Ponomarev, P. Lindh, and J. Pyrhonen, "Effect of slot-and-pole combination on the leakage inductance and the performance of tooth-coil permanent-magnet synchronous machines," IEEE Trans. Ind. Electron., vol. 60 , no. 10 , pp. 4310-4317, Oct. 2013.

[11] F. Wu, P. Zheng, L. Cheng, and C. Zhou, "Analysis and experimental evaluation of harmonic leakage inductance for polyphase pm machines having close slot and pole combinations," IEEE Trans. Magn., vol. 51, no. 11, pp. 1-4, Nov. 2015.

[12] J. F. Gieras, C. Wang, C. S. L. Joseph, and N. Ertugrul, "Analytical prediction of noise of magnetic origin produced by permanent magnet brushless motors," in Proc. IEEE Int. Elect. Mach. Drives Conf., May 2007, vol. 1, pp. 148-152.

[13] J. Islam, D. Svechkarenko, R. Chin, A. Szucs, J. Mantere, and R. Sakki, "Cogging torque and vibration analysis of a direct-driven PM wind generator with concentrated and distributed windings," in Proc. Int. Conf. Elect. Mach. Syst., Oct. 2012, pp. 1-6.

[14] J. Goss, D. Staton, R. Wrobel, and P. Mellor, "Brushless AC interiorpermanent magnet motor design: Comparison of slot/pole combinations and distributed vs. concentrated windings," in Proc. IEEE Energy Convers. Congr. Expo., Sep. 2013, pp. 1213-1219.

[15] M. Valavi, A. Nysveen, and R. Nilssen, "Influence of slot harmonics on radial magnetic forces in low-speed pm machine with concentrated windings," in Proc. Int. Conf. Elect. Mach. Syst., Oct. 2013, pp. 535-538.

[16] S.-K. Lee, G.-H. Kang, and J. Hur, "Finite element computation of magnetic vibration sources in $100 \mathrm{~kW}$ two fractional-slot interior permanent magnet machines for ship," IEEE Trans. Magn., vol. 48, no. 2, pp. 867870, Feb. 2012.

[17] H. Yang and Y. Chen, "Influence of radial force harmonics with low mode number on electromagnetic vibration of PMSM," IEEE Trans. Energy Convers., vol. 29, no. 1, pp. 38-45, Mar. 2014.

[18] G. Dajaku, H. Hofmann, F. Hetemi, X. Dajaku, W. Xie, and D. Gerling, "Comparison of two different IPM traction machines with concentrated winding," IEEE Trans. Ind. Electron., vol. 63, no. 7, pp. 4137-4149, Jul. 2016.

[19] B. Kaku, I. Miyashita, and S. Sone, "A novel prediction method of acoustic magnetic noise based on induction motor's NHCC function," IEEE Trans. Ind. Electron., vol. 46, no. 2, pp. 398-406, Apr. 1999.

[20] W. C. Lo, C. C. Chan, Z. Q. Zhu, L. Xu, D. Howe, and K. T. Chau, "Acoustic noise radiated by PWM-controlled induction machine drives," IEEE Trans. Ind. Electron., vol. 47, no. 4, pp. 880-889, Aug. 2000.

[21] A. C. Binojkumar, B. Saritha, and G. Narayanan, "Acoustic noise characterization of space-vector modulated induction motor drivesAn experimental approach," IEEE Trans. Ind. Electron., vol. 62, no. 6, pp. 3362-3371, Jun. 2015
[22] W. Liang, P. C. K. Luk, and W. Fei, "Analytical investigation of sideband electromagnetic vibration in integral-slot PMSM drive with SVPWM technique," IEEE Trans. Power Electron., vol. 32, no. 6, pp. 4785-4795, Jun. 2017.

[23] W. Liang, J. Wang, P. C. K. Luk, W. Fang, and W. Fei, "Analytical modeling of current harmonic components in PMSM drive with voltage-source inverter by SVPWM technique," IEEE Trans. Energy Convers., vol. 29, no. 3, pp. 673-680, Sep. 2014

[24] W. Liang, W. Fei, and P. C. K. Luk, "An improved sideband current harmonic model of interior PMSM drive by considering magnetic saturation and cross-coupling effects," IEEE Trans. Ind. Electron., vol. 63, no. 7, pp. 4097-4104, Jul. 2016.

[25] J. F. Gieras, C. Wang, and J. C. Lai, Noise of Polyphase Electric Motors (ser. Electrical and Computer Engineering). Boca Raton, FL, USA: CRC Press, 2005.

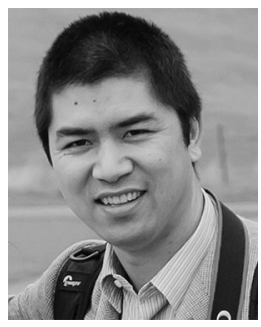

Wenyi Liang was born in Zhejiang, China, 1982. He received the B.Eng. and M.Eng. degrees in electrical engineering from Zhejiang University, Hangzhou, China, in 2004 and 2006, respectively.

From 2006 to 2014, he was a Development Engineer with Hangzhou Easitech Corporation, Hangzhou. He is currently a Research Fellow with the Power Engineering Centre, Cranfield University, Cranfield, U.K. His current research interests include design, analysis, and applications of electric machines and drives.

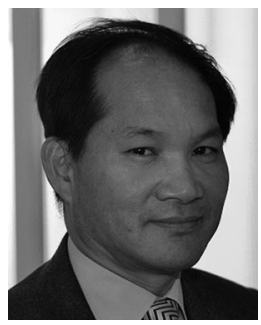

Patrick Chi-Kwong Luk (M'92-SM'08) was born in Hong Kong. He received the High Diploma with merits (B.Sc.) in electrical engineering from Hong Kong Polytechnic University, Hung Hom, Kowloon, Hong Kong, in 1983, the M.Phil. degree in electrical engineering from Sheffield University, Sheffield, U.K., in 1989, and the Ph.D. degree in electrical engineering from the University of South Wales, Pontypridd, U.K., in 1992.

He started his career in industry as an Engineer Trainee between 1981 and 1983 at GEC (Hong Kong) and then after graduation as an Applications Engineer at Polytek Engineering Co. (Hong Kong) In 1986, he was a Senior Researcher with the Industrial Centre, PolyU. Since 1988, he had held academic positions at the University of South Wales, Robert Gordon University, Aberdeen, U.K., and the University of Hertfordshire, U.K. In 2002, he joined Cranfield University, Bedford, U.K., where he is a Chair Professor in electrical engineering and the Head of the Electric Power and Drives Group, Power Engineering Centre. He has authored more than 170 publications in electric machines, power electronics, and energy conversion systems. His current research interests include electrical drives, renewable energy systems, and high-frequency power electronics.

Dr. Luk has held chair positions in the IEEE UK\&I Power Electronics/Industrial Electronics Chapters and is currently the Chair for IEEE UK\&I Professional Communications.

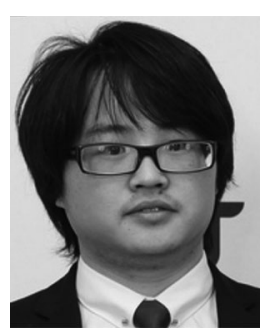

Weizhong Fei (M'12) was born in Zhejiang, China, 1981. He received the B.Eng. and M.Eng. degrees from Zhejiang University, Hangzhou, China, in 2004 and 2006, respectively, and the Ph.D. degree from Cranfield University, Shrivenham, U.K., in 2010, all in electrical engineering.

From 2011 to 2012, he was a Research Associate with the University of Sheffield, Sheffield, U.K. Since 2012, he has been with Cranfield University, Cranfield, U.K., where he is currently a Lecturer in Clean Energy Technology. His current research interests include design and applications of high-efficient energy conversion systems. 